\author{
XiaoFei $\mathrm{Wu}^{1, *}$ \\ Qiong Wang ${ }^{2, *}$ \\ Zhixian Wang ${ }^{3}$ \\ Xian Zhao ${ }^{1}$ \\ Xiaojing $\mathrm{Xu}^{4}$
}

'Department of Urology, Taikang Tongji (Wuhan) Hospital, Wuhan, 430050,

People's Republic of China; ${ }^{2}$ Department of Plastic Surgery, Wuhan One Plus One Cosmetic Hospital, Wuhan, 430030, People's Republic of China; ${ }^{3}$ Department of Urology, Tongji Hospital, Tongji Medical College, Huazhong University of Science and Technology, Wuhan, 430030, People's Republic of China; ${ }^{4}$ Department of Urology, Wuhan Hospital of Integrated Traditional Chinese and Western Medicine, Wuhan, 430022, People's Republic of China

*These authors contributed equally to this work
Correspondence: Xian Zhao Department of Urology, Taikang Tongji (Wuhan) Hospital, Wuhan, 430050, People's Republic of China Email zhaornm@।63.com

\section{Xiaojing Xu}

Department of Urology, Wuhan Hospital of Integrated Traditional Chinese and

Western Medicine, Wuhan, 430022,

People's Republic of China

Email xxj365zx@163.com
Objective: T3a renal cell carcinoma (RCC) did not consider tumor size and different extrarenal invasion patterns in the current TNM staging system. Here, we want to investigate the association of survival outcomes with different extrarenal invasion patterns and tumor size of T3a RCC.

Methods: We identified T3a RCC patients from the Surveillance, Epidemiology, and End Results database in 2004-2015. The extrarenal invasion patterns included renal vein invasion, renal sinus/peri-sinus fat invasion, or perinephric fat invasion. Cox proportional hazards models and Fine and Gray models were used to estimate overall survival (OS) and cancerspecific survival (CSS), and the hazard ratios (HRs) with 95\% confidence intervals (CIs) were calculated. $\mathrm{C}$-index is used to evaluate the predictive ability of the model. Restricted cubic splines were used to estimate the HRs of tumor size on the risk of OS and CSS.

Results: In total, 4834 T3a RCC patients were included in the analysis. Of them, 1403 (29\%) present isolated extrarenal invasion pattern, while 1403 (71\%) were any combined invasion pattern, which was associated with a higher risk of lymph-node/distant metastasis and a worse OS and CSS compared with isolated extrarenal invasion pattern, but a comparable CSS and OS were observed between each isolated invasion pattern. In restricted cubic splines, a non-linear shape was observed for the association between the tumor size and OS and CSS, compared with the smallest tumor size group $(\leq 4 \mathrm{~cm})$, the adjusted HR of the largest tumor size group $(\geq 10 \mathrm{~cm})$ was 1.59 for all-cause mortality, and 2.27 for tumor-specific mortality, respectively. However, in a cohort of T3a RCC with a combined invasion pattern, tumor size is not an independent risk factor for prognosis. Finally, the model added two covariates of tumor size and invasion patterns that could improve the predictive ability of OS and CSS for T3a patients (c-index: $+1.2 \%,+1.3 \%$, respectively).

Conclusion: Tumor size and extrarenal invasion type are valid parameters of the OS and CSS for T3a RCC patients and need to be considered for the next generation of the T-stage system.

Keywords: renal cell carcinoma, tumor size, extrarenal fat extension, T3a stage, prognosis, TNM

\section{Introduction}

Since the publishing of the first staging manual in 1977 by the American Joint Committee on Cancer (AJCC), the Tumor-Node-Metastasis (TNM) staging system has been used globally for decades as a means of cancer classification of prognosis. Within the staging system prognosis, differing cancer stages were focused on greatly. ${ }^{1}$ Currently, the $\mathrm{T}$ stage in renal cell carcinoma (RCC) is determined by 
extrarenal invasion and tumor size. ${ }^{2}$ Despite great revision across the latest editions TNM stage system, T3a faces possible prognostic discrimination due to disregard of differing extension patterns as well as tumor size.,

Prior studies suggest that extension patterns should not be a prognosticator of cancer-specific mortality. ${ }^{5-7}$ In contrast, other clinical centers showed that RCC with invasion of renal sinus/perisinus fat (RS/PSFI) or renal vein invasion (RVI) alone had a poorer prognosis than having perinephric fat invasion (PNFI) present. ${ }^{8-11}$ It was observed that any combination of PNFI, RS/PSFI, and RVI variants resulted in a worse prognosis. ${ }^{5,12-15}$ Besides, tumor size should be considered in T3a RCC staging to have a better prognostic analysis. ${ }^{4,16-19}$ Some publications reported a grouping separation at $7 \mathrm{~cm}$ may greatly reduce prognostic discrimination. ${ }^{4,18}$ BrookmanMay et $\mathrm{al}^{17}$ proposed that if a $7 \mathrm{~cm}$ cutoff were to be established for tumor size within the TNM classification system as well as combining T2-sized based subgroups could raise the predictive accuracy for prognosis. However, other researchers found that the T3a RCC significantly decreased the prognosis outcomes across all tumor sizes in $\mathrm{RCC}^{20}$ In general, the current staging system for stage T3a is controversial, and the evidence is limited. Whether it is necessary to consider the size of the tumor and the patterns of extrarenal invasion in T3a RCC for further stratification is necessary for further study.

Given there are controversies regarding the cancer special survival (CSS) in T3a RCC with different invasion patterns and tumor size, this study aimed to assess the implications of different patterns of extrarenal invasion and tumor size concerning patient prognosis for $\mathrm{T} 1-3 \mathrm{a}$ RCC to provide clinicians a benchmark for counseling for RCC patients. Here, we use a large national cancer registry (Surveillance, Epidemiology, and End Results [SEER]) for evaluating the prognosis of different patterns of extrarenal fat extension and tumor size in T3a RCC.

\section{Patients and Methods}

\section{Data Sources and Study Population}

The (SEER)-18 database (https://seer.cancer.gov/) was accessed for patients $\geq 18$ years old having been diagnosed with RCC in 2004-2015. All RCC diagnoses were confirmed by histology and not by autopsy or death certification. We considered patient data where T3a RCC was the primary malignancy with surgical treatment of partial nephrectomy ( $\mathrm{PN})$ and radical nephrectomy (RN); In the past, if T3a RCCs found preoperatively, surgeons generally recommended $\mathrm{RN}$. However, there are also many reports in the literature that $\mathrm{PN}$ is feasible in T3a RCC patients. ${ }^{21}$ In addition, PN surgery is sometimes necessary for clinical practice, such as solitary kidney tumors, patients with chronic kidney diseases, etc.; therefore, in our present study, we also included patients who performed PN. The stage of T3a was based on the SEER adjusted AJCC TNM stage corresponding years at diagnosis. Patient data sets were excluded based on the following criteria: information of invasion type was missed, tumor size $>20 \mathrm{~cm}$, missing information, and patients with an unavailable, missing, or invalid SEER cause of death record. This study was approved by the Ethics Review Board of Taikang Tongji (Wuhan) Hospital based on the Declaration of Helsinki.

\section{Analysis Variables and Outcomes}

Demographic variables for patients included diagnosis age, sex (male vs female), and race (white vs other). Tumor variables included tumor histologic cell type (clear cell carcinoma vs non-clear cell carcinoma), Fuhrman grade (I/II vs III/IV), sarcomatoid differentiation (yes vs no), extrarenal invasion patterns (RVI, PNFI, RS/ PSFI, and any combined invasion pattern), tumor size, involvement of regional lymph node ( $\mathrm{N} 0 / \mathrm{X}$ vs $\mathrm{N} 1)$, and distant metastasis (M0/x vs M1) diseases. In our study, tumor size is classified according to the current T1 and T2 stage classification standards with cutoff points of $4 \mathrm{~cm}$, $7 \mathrm{~cm}$, and $10 \mathrm{~cm}(\mathrm{~T} 3 \mathrm{a} \leq 4 \mathrm{~cm}, \mathrm{~T} 3 \mathrm{a} 4-7 \mathrm{~cm}, \mathrm{~T} 3 \mathrm{a} 7-10 \mathrm{~cm}$, and T3a $>10 \mathrm{~cm}$ ). Besides, surgical treatments of PN and RN were included as covariables for analysis. Here, we defined the RVI as tumors of T3b in the 6th edition AJCC Cancer Staging Manual (Tumor grossly extends into the renal vein or its segmental (muscle-containing) branches or vena cava below the diaphragm) was changed into T3a in 7th edition AJCC Cancer Staging Manual (renal vein or its segmental (muscle-containing) branch involvement or perirenal fat invasion). Alone invasion (AI) and combined invasion (CBI) were defined according to extrarenal fat invasion patterns in T3a RCC. AI included RVI alone, PNFI alone, and RS/PSFI alone; whereas, CBI included RVI plus PNFI, RVI plus RS/ PSFI, PNFI plus RS/PSFI, and RVI plus PNFI plus RS/ PSFI. Overall survival (OS) and cancer specific survival (CSS) were the primary study outcomes in our study. 


\section{Statistical Analyses}

Differences between the distributions of patient demographics and tumor characteristics were compared, continuous variables and categorical variables were presented as median (interquartile range, IQR) and $\mathrm{N}(\%)$, and compared with the Mann-Whitney $U$-test and Chisquare test, respectively. The trends of the baseline characteristics across different tumor size groups ( $\mathrm{T} 3 \mathrm{a} \leq 4 \mathrm{~cm}$, T3a $4-7 \mathrm{~cm}$, T3a $7-10 \mathrm{~cm}$, and T3a $\geq 10 \mathrm{~cm}$ ) were assessed by using logistic or linear regression analysis. The OS based on the invasion types and different tumor size groups were compared via the log-rank statistic KaplanMeier method for OS; The cumulative incidence function curve of cancer-specific mortality was plotted and compared by Gray's test. Univariate and multivariate Cox proportional hazards models and Fine and Gray models were used to estimate overall survival (OS) and cancerspecific survival (CSS) and the hazard ratios (HRs) or sub-distribution hazard ratio (sHRs) with $95 \%$ confidence intervals (CIs) were calculated to assess the effect of covariables on OS and CSS. C-index is used to evaluate the predictive ability of the model. Multivariate logistic regression analysis was used to evaluate independent variables associated with the different types of fat invasion in T3a patients and to assess the predictive effects of extrarenal extension sites on $\mathrm{N} 1$ and M1, odds ratio (OR) with 95\% CIs for all risk factors. To assess the HRs of tumor size on the risk of OS and CSS, the restricted cubic splines were used to show the shape of these associations with four knots placed at the 5th, 35th, 65th, and 95th percentiles of tumor size. The non-linearity test is carried out through the likelihood ratio test, which compares the log-likelihood of the model containing the linear term with the model containing the cubic spline term. Since $\mathrm{RN}$ is still a standard treatment for clinical T3a RCC, but in our present study, patients treated with PN were also included in the analysis, which might affect survival outcomes as a selection bias, therefore we also conducted a sensitivity analysis that excluded the cohort of patients performed PN. In addition, because $\mathrm{Nx} / \mathrm{Mx}$ can include $\mathrm{N} 1 / \mathrm{M} 1$, and there is a bias for analysis of risk factors, so another sensitive analysis which classified $\mathrm{N}$ and $\mathrm{M}$ stage as N0, $\mathrm{N}_{\mathrm{X}}$, and $\mathrm{N} 1$ and M0, MX, and M1 in the multivariate survival analysis. All p-values were two-sided, and any $<0.05$ were deemed statistically significant. $\mathrm{R}$ statistical package (v.4.1.0) was used to assess all analyses.

\section{Results}

A total of $4834 \mathrm{~T} 3 \mathrm{a}$ RCC patients were included in the analysis (Table 1). There were 1403 (29\%) T3a RCCs present CBI pattern, and 3431 (71.0\%) tumors present AI pattern, most of them were RVI $(28.7 \%)$, while the PNFI and RS/PSFI accounted $28.2 \%$ and $14.0 \%$, respectively. Compared to T3a RCC with AI pattern, tumors with CBI pattern were more likely to have clear cell RCC $(92.9 \%$ vs $87.3 \%$ ), Fuhrman grade III-IV (75.1\% vs $58.7 \%$ ), sarcomatoid differentiation $(11.3 \%$ vs $4.95 \%)$, tumor size $>10 \mathrm{~cm}(36.8 \%$ vs $22.2 \%)$, N1 (13.5\% vs $5.48 \%)$, and M1 (26.7\% vs $12.8 \%)$; and more radical nephrectomy were performed $(96.0 \%$ vs $86.3 \%)$; while age, gender, and race showed no significant differences between the AI pattern and CBI pattern groups Table 1).

Besides, we found that older age, clear cell RCC, higher Fuhrman grade, sarcomatoid differentiation, and larger tumor size were independently associated with a higher risk of the characteristics of CBI pattern present in T3a RCC (Supplementary Table 1). Supplementary Table 2 shows that T3a RCC with CBI pattern had a higher risk of $\mathrm{N} 1$ diseases and $\mathrm{M} 1$ diseases compared to tumors with AI pattern (OR $=1.960$ and 1.69, respectively). While RS/PSFI had a higher risk for N1 compared to RVI and PNFI alone (OR $=1.57$ and 1.48 , respectively), PNFI and RS/PSFI alone had a higher chance of 1.58- and 1.41-fold for M1 diseases than RVI alone, and RS/PSFI did not significantly increase the risk of M1 diseases compared to PNFI alone (OR $=0.988,95 \%$ CI: 0.74 $1.31, \mathrm{p}=0.933)$.

The median of tumor size was $7.5 \mathrm{~cm}$ (IQR: $5.2 \mathrm{~cm}-$ $10.0 \mathrm{~cm}$ ) (Table 2). Patients with larger T3a RCCs diameter were younger age, more likely to be clear-cell carcinoma and sarcomatoid differentiation, higher Fuhrman grade, preferred to radical nephrectomy, the higher chance of $\mathrm{N} 1$ diseases and M1 diseases, and more present with CBI pattern. These results were consistent results that come from the cohort of AI (Supplementary Table 3) and CBI pattern (Supplementary Table 4).

The T3a RCC with CBI pattern had a lower OS and CSS than T3a RCC with AI invasion (Figure 1A and B). When the demographic and clinical features were adjusted, CBI had a higher risk of OS (adjusted HR: $1.19 ; 95 \% \mathrm{CI}$ : 1.04-1.35; $\mathrm{p}=0.01$ ) and CSS (adjusted HR: $1.18 ; 95 \% \mathrm{CI}$ : $1.02-1.37 ; \mathrm{p}=0.03$ ) than AI. While the invasion type of RVI, PNFI, and RS/PSFI alone did not show significant 
Table I Baseline Characteristics According to Extrarenal Extension Pattern in the Cohort of T3a Renal Cell Carcinoma

\begin{tabular}{|c|c|c|c|c|}
\hline & [ALL] $N=4834$ & Alone Invasion N=343 I & Combined Invasion $N=\mid 403$ & p-value \\
\hline Age at diagnosis. Year & $63.0[55.0 ; 71.0]$ & $63.0[55.0 ; 70.0]$ & $63.0[55.0 ; 71.0]$ & 0.26 \\
\hline Sex: & & & & 0.194 \\
\hline Female & 1476 (30.5\%) & 1067 (31.1\%) & $409(29.2 \%)$ & \\
\hline Male & 3358 (69.5\%) & 2364 (68.9\%) & 994 (70.8\%) & \\
\hline Race: & & & & 0.624 \\
\hline White & 4208 (87.1\%) & 2981 (86.9\%) & 1227 (87.5\%) & \\
\hline Other & $626(12.9 \%)$ & $450(13.1 \%)$ & $176(12.5 \%)$ & \\
\hline Histology: & & & & $<0.001$ \\
\hline Clear cell carcinoma & 4297 (88.9\%) & 2994 (87.3\%) & 1303 (92.9\%) & \\
\hline Non-clear cell carcinoma & 537 (11.1\%) & 437 (I2.7\%) & $100(7.13 \%)$ & \\
\hline Fuhrman grade: & & & & $<0.001$ \\
\hline $\mathrm{I} / \mathrm{II}$ & 1766 (36.5\%) & $14 \mid 7$ (4I.3\%) & 349 (24.9\%) & \\
\hline III/IV & 3068 (63.5\%) & $2014(58.7 \%)$ & 1054 (75.1\%) & \\
\hline Sarcomatoid differentiation: & & & & $<0.001$ \\
\hline Yes & $329(6.81 \%)$ & 170 (4.95\%) & $159(11.3 \%)$ & \\
\hline No & 4505 (93.2\%) & 3261 (95.0\%) & 1244 (88.7\%) & \\
\hline Size of tumor, $\mathrm{cm}$ & $7.50[5.20 ; 10.0]$ & $7.00[4.90 ; 9.50]$ & $8.50[6.50 ; 11.0]$ & $<0.001$ \\
\hline Tumor size group & & & & $<0.001$ \\
\hline $\mathrm{T} 3 \mathrm{a} 4 \mathrm{~cm}$ & 667 (13.8\%) & 587 (17.1\%) & $80(5.70 \%)$ & \\
\hline T3a $4-7 \mathrm{~cm}$ & I 584 (32.8\%) & II 98 (34.9\%) & $386(27.5 \%)$ & \\
\hline Т3a $7-10 \mathrm{~cm}$ & I 304 (27.0\%) & $883(25.7 \%)$ & $421(30.0 \%)$ & \\
\hline $\mathrm{T} 3 \mathrm{a} \geq 10 \mathrm{~cm}$ & 1279 (26.5\%) & $763(22.2 \%)$ & $516(36.8 \%)$ & \\
\hline Surgery: & & & & $<0.001$ \\
\hline Partial nephrectomy & $526(10.9 \%)$ & $470(13.7 \%)$ & 56 (3.99\%) & \\
\hline Radical nephrectomy & 4308 (89.1\%) & 2961 (86.3\%) & 1347 (96.0\%) & \\
\hline N stage: & & & & $<0.001$ \\
\hline $\mathrm{N} 0 / \mathrm{x}$ & 4456 (92.2\%) & $3243(94.5 \%)$ & $1213(86.5 \%)$ & \\
\hline NI & 378 (7.82\%) & $188(5.48 \%)$ & $190(13.5 \%)$ & \\
\hline M stage: & & & & $<0.001$ \\
\hline $\mathrm{M} 0 / \mathrm{x}$ & 4022 (83.2\%) & $2993(87.2 \%)$ & $1029(73.3 \%)$ & \\
\hline MI & $812(16.8 \%)$ & $438(12.8 \%)$ & $374(26.7 \%)$ & \\
\hline Invasion types 2: & & & & l \\
\hline Combined invasion & I403 (29.0\%) & $0(0.00 \%)$ & 1403 (100\%) & \\
\hline Perinephric fat invasion & 1363 (28.2\%) & 1363 (39.7\%) & $0(0.00 \%)$ & \\
\hline Renal sinus/peri-sinus fat invasion & 679 (14.0\%) & 679 (19.8\%) & $0(0.00 \%)$ & \\
\hline Renal vein invasion & 1389 (28.7\%) & 1389 (40.5\%) & $0(0.00 \%)$ & \\
\hline
\end{tabular}

Note: Data are shown as median (IQR) or frequency.

Abbreviation: IQR: interquartile range.

differences for OS and CSS, but all had a lower risk of mortality than the CBI pattern (Figure $1 \mathrm{C}$ and D).

Figure 2 presents the non-linear trends in the associations between tumor size and the all-cause of mortality and tumor-specific mortality from the restricted cubic spline analyses. The risk of all-cause mortality was relatively flat in T3a RCCs of smaller size but increased rapidly between around $5 \mathrm{~cm}$ and $10 \mathrm{~cm}$ and then plateaued thereafter (Figure 2A-C). However, in the cohort of T3a RCC present $\mathrm{CBI}$ pattern, the risk of all-cause mortality was increased rapidly until $10 \mathrm{~cm}$ and then plateaued thereafter (Figure 2B). A similar non-linear shape was also seen for the cancer-specific mortality (Figure 2D-F). A sensitivity analysis that excluded the cohort of patients who performed PN also observed a consistent result (Supplementary Figure 1). The individual had a larger T3a RCCs tumor size classification, the worse OS and CSS experienced, and this trend is still present in the cohort of AI patterns and CBI pattern cohort (Figure 3). However, after adjusting other covariables, a larger tumor 
Table 2 Baseline Characteristics According to Size Classification

\begin{tabular}{|c|c|c|c|c|c|c|c|}
\hline & \multirow{2}{*}{$\begin{array}{l}\text { All Cohort } \\
N=4834\end{array}$} & \multicolumn{4}{|c|}{ Size Classification } & \multirow[t]{2}{*}{ p-value } & \multirow{2}{*}{$\begin{array}{l}P \text { for } \\
\text { Trend }\end{array}$} \\
\hline & & $\begin{array}{l}T 3 a \leq 4 \mathrm{~cm} \\
N=667\end{array}$ & $\begin{array}{l}T 3 a 4-7 \mathrm{~cm} \\
N=1584\end{array}$ & $\begin{array}{l}\text { T3a } 7-10 \mathrm{~cm} \\
N=1304\end{array}$ & $\begin{array}{l}T 3 a \geq 10 \mathrm{~cm} \\
N=1279\end{array}$ & & \\
\hline Age at diagnosis. Year & $63.0[55.0 ; 71.0]$ & $64.0[55.0 ; 71.0]$ & $64.0[56.0 ; 72.0]$ & $63.0[55.0 ; 70.0]$ & $60.0[53.0 ; 68.0]$ & $<0.001$ & $<0.001$ \\
\hline Sex: & & & & & & 0.011 & 0.44 \\
\hline Female & 1476 (30.5\%) & 231 (34.6\%) & 495 (31.2\%) & 359 (27.5\%) & 391 (30.6\%) & & \\
\hline Male & 3358 (69.5\%) & $436(65.4 \%)$ & $1089(68.8 \%)$ & 945 (72.5\%) & 888 (69.4\%) & & \\
\hline Race: & & & & & & 0.184 & 0.268 \\
\hline White & 4208 (87.1\%) & 575 (86.2\%) & 1399 (88.3\%) & II 38 (87.3\%) & $1096(85.7 \%)$ & & \\
\hline Other & $626(12.9 \%)$ & $92(13.8 \%)$ & 185 (II.7\%) & $166(12.7 \%)$ & $183(14.3 \%)$ & & \\
\hline Histology: & & & & & & $<0.001$ & $<0.001$ \\
\hline Clear cell carcinoma & 4297 (88.9\%) & 515 (77.2\%) & 1422 (89.8\%) & $1215(93.2 \%)$ & II 45 (89.5\%) & & \\
\hline $\begin{array}{l}\text { Non-clear cell } \\
\text { carcinoma }\end{array}$ & 537 (II.1\%) & I52 (22.8\%) & $162(10.2 \%)$ & 89 (6.83\%) & $134(10.5 \%)$ & & \\
\hline Fuhrman grade: & & & & & & $<0.001$ & $<0.001$ \\
\hline $\mathrm{I} / \mathrm{II}$ & 1766 (36.5\%) & 390 (58.5\%) & 669 (42.2\%) & 426 (32.7\%) & 281 (22.0\%) & & \\
\hline III/IV & 3068 (63.5\%) & 277 (4I.5\%) & $915(57.8 \%)$ & $878(67.3 \%)$ & 998 (78.0\%) & & \\
\hline $\begin{array}{l}\text { Sarcomatoid } \\
\text { differentiation: }\end{array}$ & & & & & & $<0.001$ & $<0.00$ I \\
\hline Yes & $329(6.81 \%)$ & $10(1.50 \%)$ & $73(4.61 \%)$ & $100(7.67 \%)$ & 146 (II.4\%) & & \\
\hline No & 4505 (93.2\%) & 657 (98.5\%) & $151 \mid(95.4 \%)$ & 1204 (92.3\%) & II 33 (88.6\%) & & \\
\hline Size of tumor. $\mathrm{cm}$ & $7.50[5.20 ; 10.0]$ & $3.20[2.50 ; 3.60]$ & $5.80[5.00 ; 6.50]$ & $8.50[7.80 ; 9.00]$ & $12.0[10.5 ; 13.5]$ & $<0.001$ & $<0.001$ \\
\hline Surgery: & & & & & & $<0.001$ & $<0.001$ \\
\hline Partial nephrectomy & $526(10.9 \%)$ & $282(42.3 \%)$ & 180 (II.4\%) & $48(3.68 \%)$ & $16(1.25 \%)$ & & \\
\hline Radical nephrectomy & 4308 (89.1\%) & 385 (57.7\%) & I 404 (88.6\%) & 1256 (96.3\%) & I 263 (98.7\%) & & \\
\hline N stage: & & & & & & $<0.001$ & $<0.001$ \\
\hline No/x & $4456(92.2 \%)$ & $652(97.8 \%)$ & I508 (95.2\%) & $1200(92.0 \%)$ & $1096(85.7 \%)$ & & \\
\hline NI & 378 (7.82\%) & $15(2.25 \%)$ & $76(4.80 \%)$ & 104 (7.98\%) & $183(14.3 \%)$ & & \\
\hline M stage: & & & & & & $<0.001$ & $<0.001$ \\
\hline $\mathrm{M} 0 / \mathrm{x}$ & 4022 (83.2\%) & 649 (97.3\%) & 1410 (89.0\%) & 1057 (8I.1\%) & 906 (70.8\%) & & \\
\hline MI & $812(16.8 \%)$ & $18(2.70 \%)$ & 174 (II.0\%) & 247 (I8.9\%) & $373(29.2 \%)$ & & \\
\hline Invasion types: & & & & & & $<0.001$ & $<0.001$ \\
\hline Alone invasion type & 3431 (7I.0\%) & 587 (88.0\%) & 1198 (75.6\%) & $883(67.7 \%)$ & 763 (59.7\%) & & \\
\hline Combined invasion & I 403 (29.0\%) & $80(12.0 \%)$ & $386(24.4 \%)$ & 421 (32.3\%) & $516(40.3 \%)$ & & \\
\hline Invasion types 2: & & & & & & $<0.001$ & I \\
\hline Combined invasion & 1403 (29.0\%) & $80(12.0 \%)$ & $386(24.4 \%)$ & 421 (32.3\%) & $516(40.3 \%)$ & & \\
\hline Perinephric fat invasion & I 363 (28.2\%) & 328 (49.2\%) & 458 (28.9\%) & $302(23.2 \%)$ & 275 (21.5\%) & & \\
\hline $\begin{array}{l}\text { Renal sinus/peri-sinus } \\
\text { fat invasion }\end{array}$ & $679(14.0 \%)$ & 119 (17.8\%) & 258 (16.3\%) & $160(12.3 \%)$ & 142 (II.1\%) & & \\
\hline Renal vein invasion & 1389 (28.7\%) & 140 (2I.0\%) & $482(30.4 \%)$ & 421 (32.3\%) & $346(27.1 \%)$ & & \\
\hline
\end{tabular}

Note: Data are shown as median (IQR) or frequency.

Abbreviation: IQR, interquartile range.

size classification showed no significant difference of OS and CSS than small classifications in the cohort of CBI pattern, although there is a trend of larger tumor size had a worse OS and CSS in CBI cohort (all $\mathrm{p}$ for trend $<0.05$ ) (Table 3). A sensitive analysis also identified such results (Supplementary Table 5).

Finally, C-index is used to reflect the predictive ability of the predictive model in T3a RCC (Table 4). We found that the model added two covariates of tumor size and invasion patterns that could improve the predictive ability of OS and CSS, the complete increase of c-index is $+1.2 \%$ and $+1.3 \%$ compared with the model not included two covariables, respectively. In addition, adding the size of T3a RCC in the AI cohort can increase the predictive ability of the model $(+1.6 \%$ for OS prediction and $+1.5 \%$ for CSS prediction), but it does not obviously improve the 


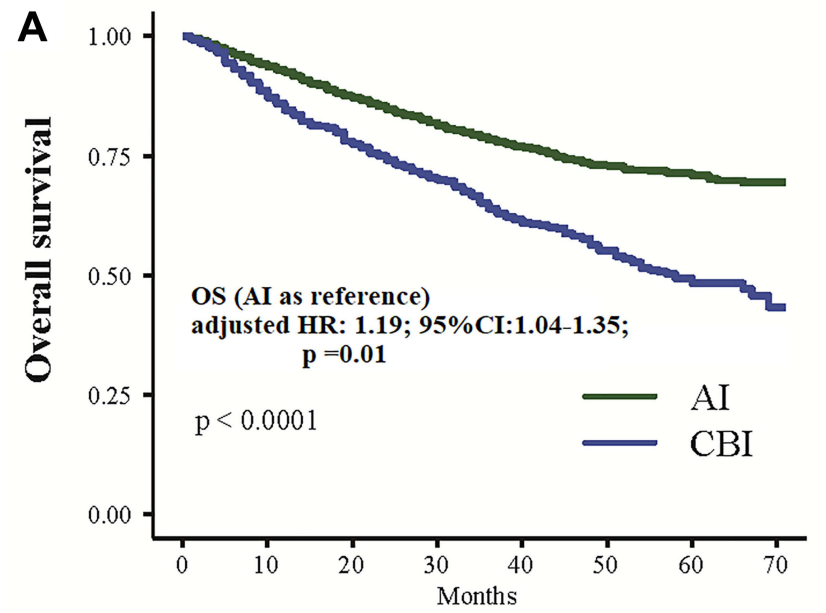

Number at risk

\begin{tabular}{|c|c|c|c|c|c|c|c|}
\hline $\mathrm{AI}-3431$ & 2660 & 1997 & 1447 & 1034 & 625 & 331 & 51 \\
\hline 1403 & 1017 & 709 & 508 & 320 & 190 & 93 & 16 \\
\hline 0 & 10 & 20 & 30 & 40 & 50 & 60 & 70 \\
\hline
\end{tabular}

C

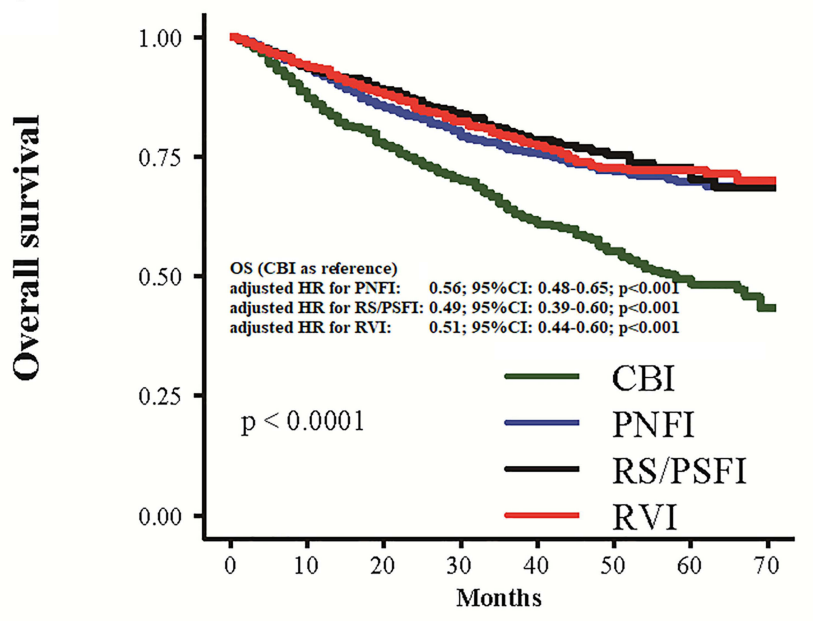

Number at risk

\begin{tabular}{|c|c|c|c|c|c|c|c|}
\hline CBI -1403 & 1017 & 709 & 508 & 320 & 190 & 93 & 16 \\
\hline NFI -1363 & 1084 & 820 & 610 & 444 & 276 & 155 & 21 \\
\hline /PSFI 1679 & 504 & 363 & 252 & 176 & 102 & 59 & 8 \\
\hline II- -1389 & 1072 & 814 & 585 & 414 & 247 & 117 & 22 \\
\hline 0 & 10 & 20 & 30 & 40 & 50 & 60 & 70 \\
\hline
\end{tabular}

B

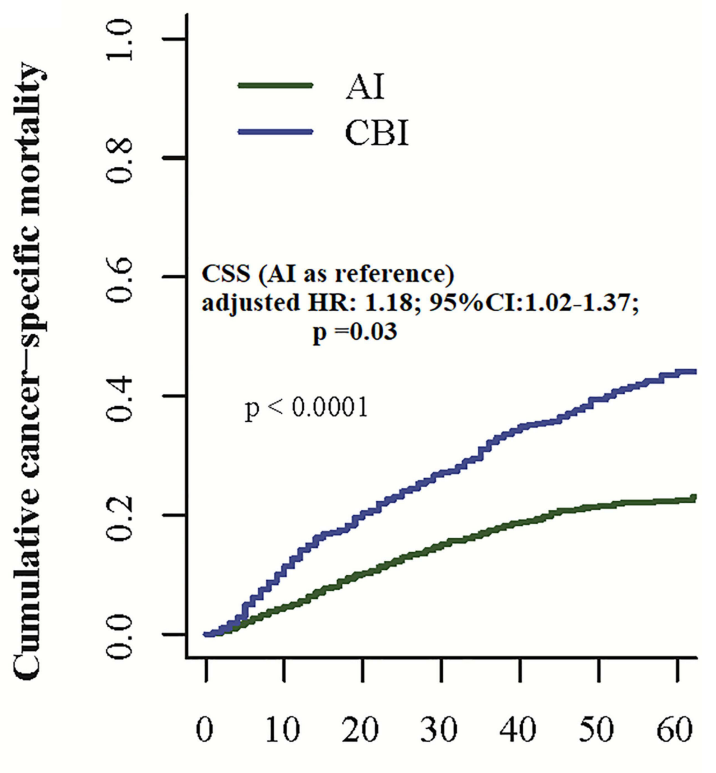

D

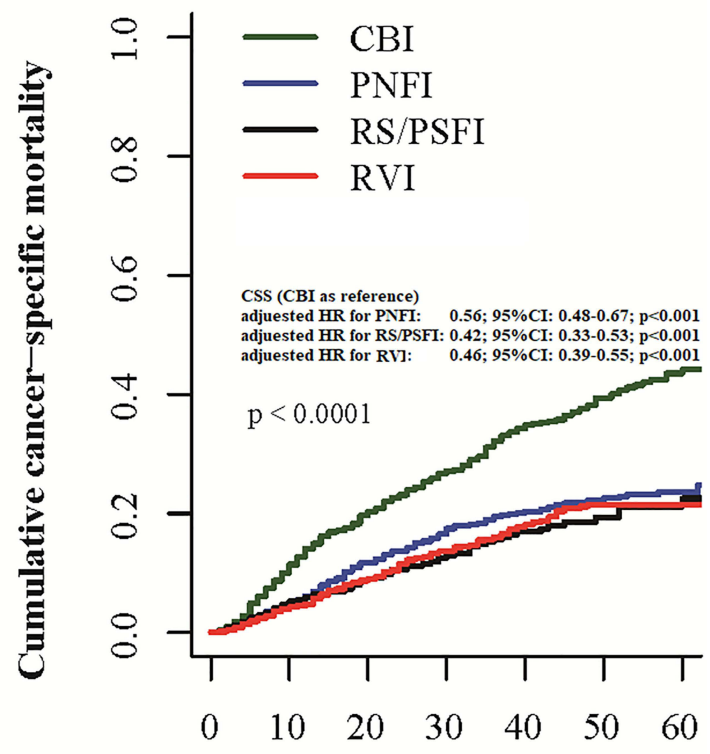

Months

Figure I Overall survival and cancer-specific mortality estimates for 4834 T3a renal cell carcinoma according to extrarenal invasion patterns. Overall survival (A) and cancer-special mortality (B) were compared between $\mathrm{CBI}$ pattern and Al pattern; Overall survival (C) and cancer-special mortality (D) were compared between $\mathrm{CBI}$ pattern, RVI pattern, PNFI pattern, and RS/PSFI pattern.

Abbreviations: RS/PSFI, medial invasion to renal sinus/perisinus fat; RVI, renal vein invasion; PNFI, perinephric fat invasion; $\mathrm{CBI}$, any combined invasion type; $\mathrm{Al}$, alone invasion.

predictive ability in the $\mathrm{CBI}$ cohort $(+0.3 \%$ for OS prediction and $+0.3 \%$ for CSS prediction).

\section{Discussion}

In the present study, we found that tumor size and extrarenal invasion patterns of T3a RCC were independent significantly associated with OS and CSS. Tumor size has a nonlinear relationship with the prognosis of T3a RCC, a larger T3a tumor size classification had a worse OS and CSS. In addition, the AI pattern experienced a better OS and CSS than the CBI pattern. What's more, we found that the model added two covariates of tumor 

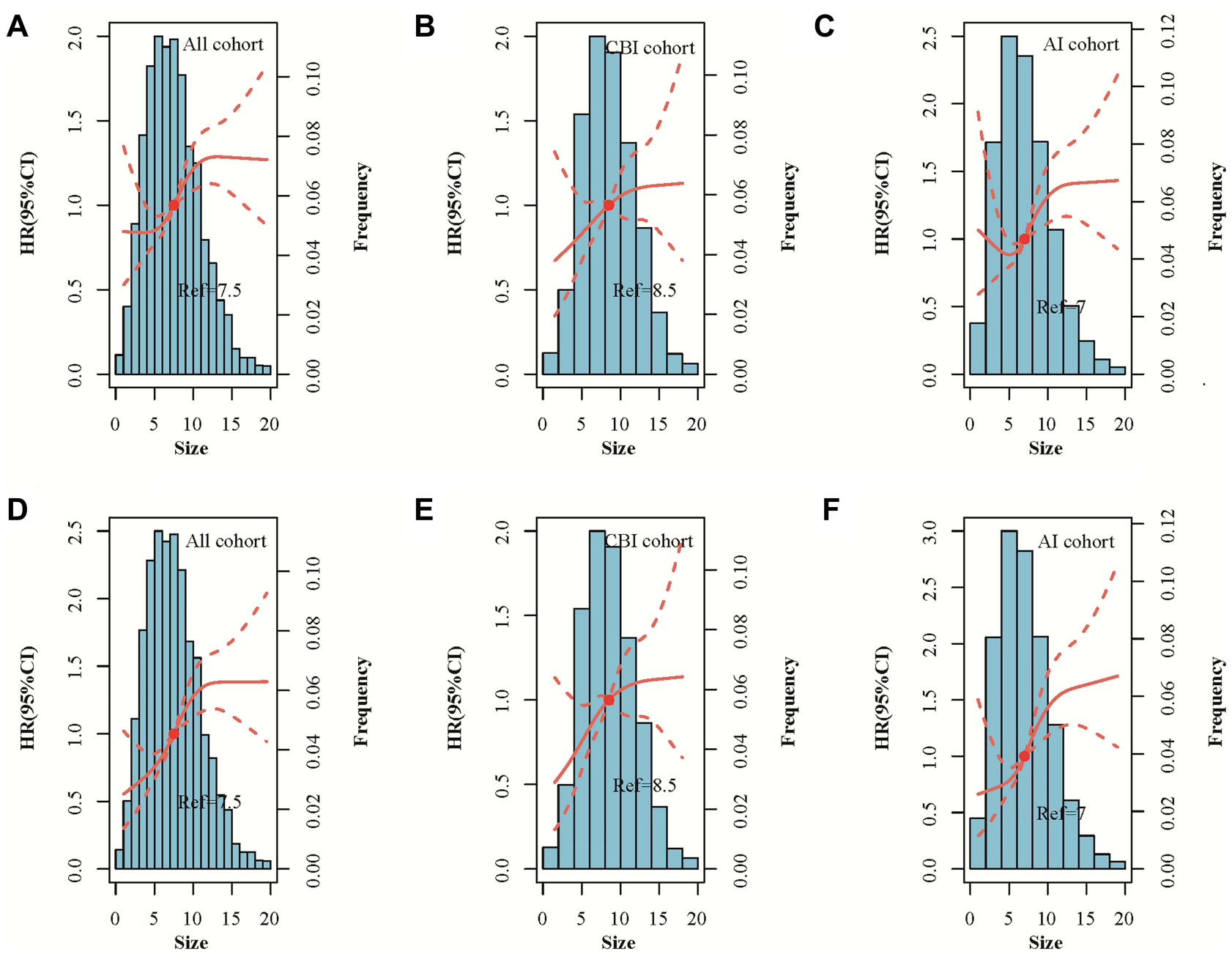

Figure 2 Restricted cubic splines for the association between tumor size and hazard risk of overall mortality and cancer-special mortality. Association between the Hazard ratio of overall mortality with tumor size in the cohort of all cases (A), CBI pattern cohort (B), and Al pattern cohort (C); and Association between the Hazard ratio of cancer-special mortality with tumor size in the cohort of all cases (D), CBI pattern cohort (E), and Al pattern cohort (F). Solid lines represent the hazard ratio (HR); dashed lines represent the $95 \% \mathrm{Cls}$. The hazard risk estimates were adjusted for age, sex, race, histology, Fuhrman grade, sarcomatoid differentiation, surgery, N/M stage, and invasion types.

Abbreviations: $\mathrm{CBI}$, any combined invasion type; $\mathrm{Al}$, alone invasion.

size and invasion patterns that could improve the predictive ability of OS and CSS than the model not including two covariables. Our present results suggested that there is still a need for further stratification regarding the current staging of T3a RCC.

As stated in the current AJCC TNM staging system for RCC, RVI, PNFI, and RS/PSFI had a similar survival outcome and was considered as one category of the T3a stage. However, it ignores the CBI pattern of T3a RCC, which has a worse prognosis than the AI pattern, besides in our present study we also found that the CBI pattern could impact the effect of tumor size on the OS and CSS; Tumor size is not an independent risk factor in T3a RCC with the invasion patterns of CBI, but in T3a RCC of the invasion patterns of $\mathrm{AI}$, tumor size is an independent risk factor for prognosis.
Some prior publications have investigated the prognosis of the extrarenal extension patterns. Part results of our present study were consistent with Shah et al. ${ }^{13}$ They observed the differing extra-renal extension prognosis within T3a RCC afflicted patients. The presence of any multiple patterns of extrarenal invasion in T3a RCCs experienced worse tumor-specific mortality after radical nephrectomy compared to the isolated presence of RVI, PNFI, or RS/PSFI. However, this study was limited to risk-stratified patients with T3aN0/ xM0 clear-cell RCC and the tumor size was not taken into consideration. Our study found that tumor size has a nonlinear relationship with the prognosis of T3a RCC through the restricted cubic spline method. And it is more rigorous than tumor size is considered as a category variable in the COX model or the competitive risk model. 
A

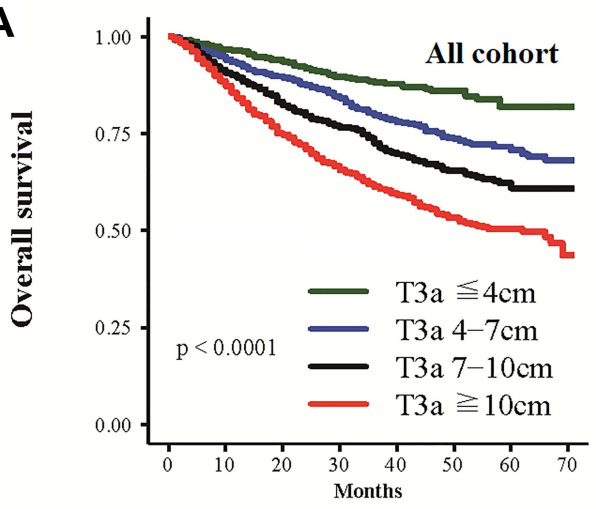

Number at risk

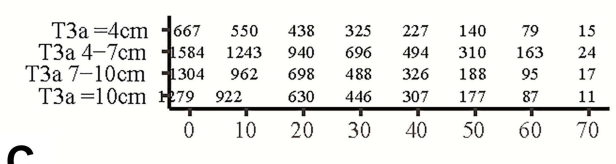

C

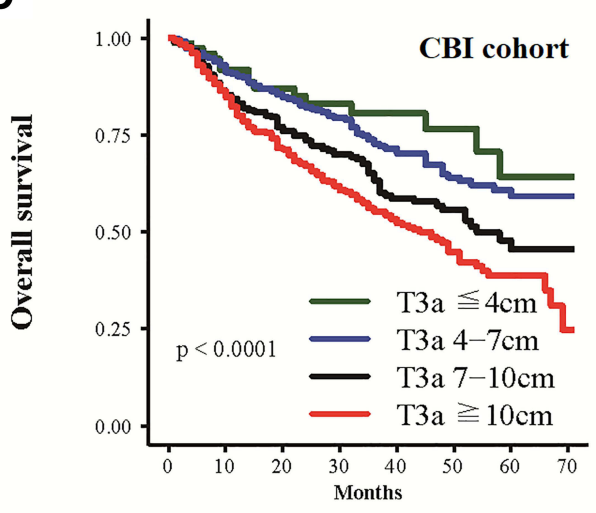

Number at risk
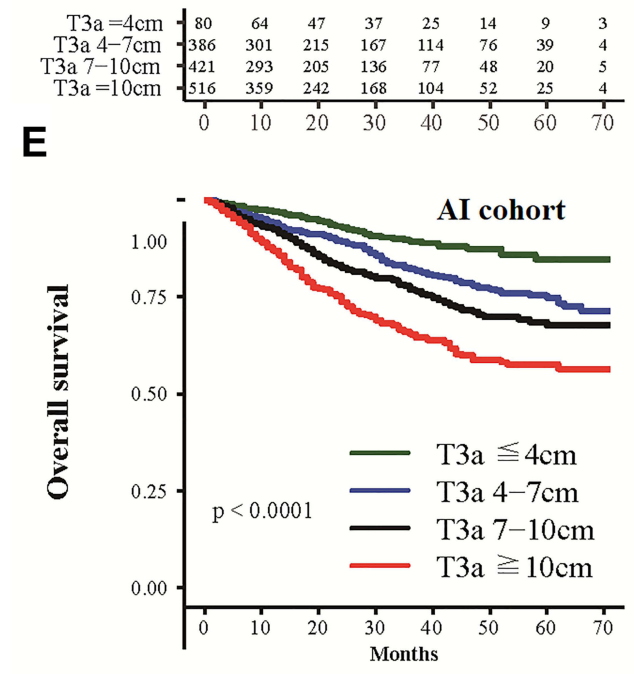

Number at risk

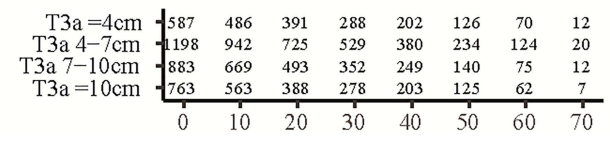

B

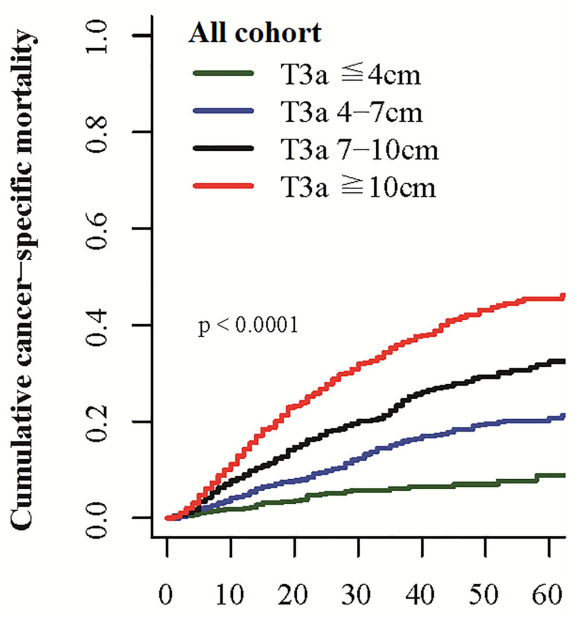

Months

D

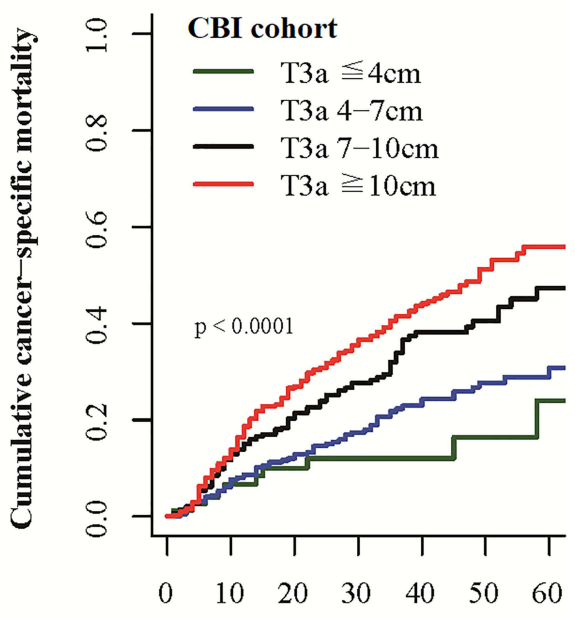

$\mathbf{F}$

Months

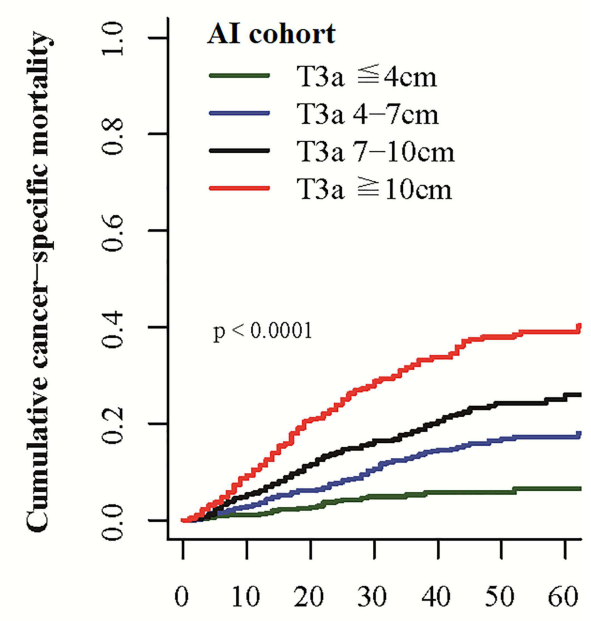

Months

Figure 3 Overall survival and cancer-specific mortality estimates for 4834 T3a renal cell carcinoma based on the tumor size category in different extrarenal invasion pattern cohorts. Overall survival (A) and cancer-special mortality (B) in the overall cohort; Overall survival (C) and cancer-special mortality (D) in CBI cohort; and Overall survival (E) and cancer-special mortality (F) in Al cohort, and RS/PSFI pattern.

Abbreviations: $\mathrm{CBI}$, any combined invasion type; $\mathrm{Al}$, alone invasion. 
Table $3 \mathrm{HRs}$ and 95\% Cls of All-Cause Mortality and Cancer-Special Mortality Based on the Tumor Size in All-, CBI-, and Al Cohort

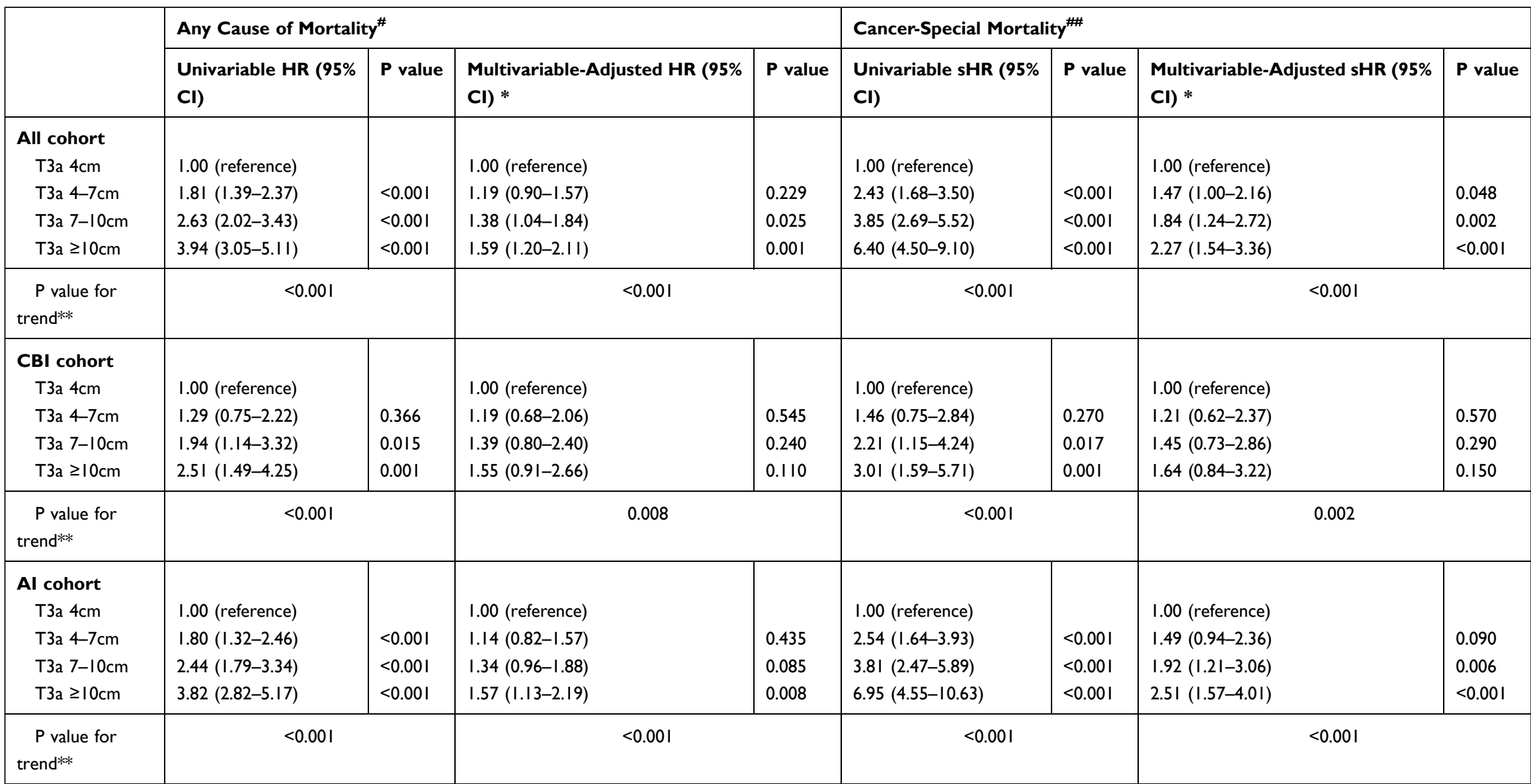

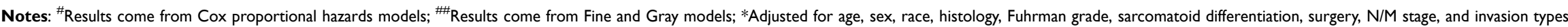
**Trend tests were conducted by treating the size classification as a continuous variable.

Abbreviations: $\mathrm{HR}$, hazard ratio; sHR, sub-distribution hazard ratio; $\mathrm{Cl}$, confidence interval; $\mathrm{CBI}$, combined invasion (any combined type of renal vein invasion, renal sinus/peri-sinus fat invasion, or perinephric fat invasion); Al, alone invasion (isolated invasion type of renal vein invasion, or renal sinus/peri-sinus fat invasion, or perinephric fat invasion) 
Table 4 The c-Index for Different Cox Models for Predicting the Overall Survival (OS) and Cancer-Special Survival (CSS) ${ }^{\#}$

\begin{tabular}{|c|c|c|c|c|c|}
\hline & & \multicolumn{2}{|l|}{ os } & \multicolumn{2}{|l|}{ CSS } \\
\hline & & C-Index & $\begin{array}{l}\text { Complete } \\
\text { Increase* }\end{array}$ & C-Index & $\begin{array}{l}\text { Complete } \\
\text { Increase* }\end{array}$ \\
\hline \multirow[t]{5}{*}{ All cohort } & & & & & \\
\hline & $\begin{array}{l}\text { Model without the characteristics of } \mathrm{T} 3 \mathrm{a} \text { tumor size and } \\
\text { invasion types }\end{array}$ & 0.776 & reference & 0.814 & reference \\
\hline & $\begin{array}{l}\text { Model with both the characteristics of T3a tumor size and } \\
\text { invasion types }\end{array}$ & 0.788 & +0.012 & 0.827 & +0.013 \\
\hline & Model with the characteristics of T3a invasion types & 0.778 & +0.002 & 0.817 & +0.003 \\
\hline & Model with the characteristics of T3a tumor size & 0.780 & +0.004 & 0.822 & +0.007 \\
\hline \multicolumn{6}{|l|}{ Al cohort } \\
\hline & Model without the characteristics of T3a tumor size & 0.768 & reference & 0.808 & reference \\
\hline & Model with the characteristics of T3a tumor size & 0.784 & +0.016 & 0.823 & +0.015 \\
\hline \multirow[t]{3}{*}{ CBI cohort } & & & & & \\
\hline & Model without the characteristics of T3a tumor size & 0.760 & reference & 0.795 & reference \\
\hline & Model with the characteristics of T3a tumor size & 0.763 & +0.003 & 0.798 & +0.003 \\
\hline
\end{tabular}

Notes: ${ }^{\#}$ The baseline model included the covariables of age, sex, race, histology, Fuhrman grade, sarcomatoid differentiation, surgery, N/M stage. ${ }^{*} T$ The c-index value of the model with tumor size and invasion method added minus the c-index value of the model without tumor size and/or invasion method.

In our study, in comparison with non-clear-cell carcinoma, clear-cell carcinoma showed a lower chance of experiencing CBI, in addition, our results were adjusted N1 diseases and M1 diseases. Similar results also come from other prior publications regarding the prognosis of different extrarenal invasion patterns of T3a RCCs. ${ }^{5,6,8-12,14,15,17,22-24}$ Kresowik et al ${ }^{12}$ noted that the prognoses of PNFI alone and RS/PSFI alone were not significantly different and T3a tumors with combinational PNFI plus RS/PSFI were associated with a worse prognosis than each alone invasion pattern. Bedke et $\mathrm{al}^{5}$ utilizing a cohort of 106 patients with T3a, in the study, 54.7\% T3a RCCs were PNFI alone, 19.8\% were RS/PSFI, and 25.5\% were PNFI plus RS/PSFI, they also illustrated similar results, T3a RCCs with CBI had a $34.1 \%$ higher risk of tumor-specific mortality than patients with either only PNFI or RS/PSFI. da Costa et $\mathrm{al}^{14}$ conducted a study illustrating a poor survival function among patients afflicted by a combination of fat invasion and RVI.

Inconsistent and controversial conclusions made by other publications may be due to study sample size and cohort inhomogeneity. Our conclusion through the large sample size data is to better verify the results of the previous small sample size research. The size of the sample size can affect the reliability of the conclusion. A study by Thompson et $\mathrm{al}^{8}$ from the Mayo Clinic center in a 212 patient population afflicted by T3a RCC found that RS/PSFI had a poor prognosis than PNFI alone; however, Shah et $\mathrm{al}^{13}$ also from the Mayo Clinic center re-examined the data excluding patients with metastatic diseases and increasing the sample size and found that the RS/PSFI and PNFI showed no significant difference in survival outcomes. Bertini et al ${ }^{9}$ conducted a study including 105 patients with T3a clear-cell RCC, it was found that T3a RCCs presenting with RS/PSFI significantly affect tumor-specific mortality, had worse tumorspecific mortality compared to PNFI in a cohort of N0/M0 diseases, but in the cohort of N1/M1 diseases, it was not associated with poor tumor-specific mortality than PNFI. Margulis et $\mathrm{al}^{6}$ including 365 patients with T3a RCC found that, in the cohort of surgically treated patients with T3a RCC, RS/PSFI and PNFI were comparable prognosticators of tumor-specific mortality but no evidence was seen showing that RS/PSFI plus PNFI had a poor 5-year survival than either of them alone. Notably, a higher prevalence of RS/ PSFI alone $(26.3 \%)$ and more than half of the patients (55.7\%) with PNFI plus RS/PSFI showed metastases, which may contradict the impact of features of extrarenal extension patterns on tumor-specific mortality.

For a T3a RCC, tumor size can be another prognostic factor. Siemer et $\mathrm{al}^{25}$ proposed that $7 \mathrm{~cm}$ as the cutoff point will improve the prognostic accuracy in the group of T3aN0/ xM0 patients. Here they suggested that patients afflicted with T3a can be categorized as such: $\leq 7 \mathrm{~cm}$ tumor is incorporated into $\mathrm{T} 1$, tumor size $>7 \mathrm{~cm}$ incorporated into T2. Thereafter, Lam et al and Brookman-May et $\mathrm{al}^{4,17}$ conducted a multi-centric study and reinforced tumor size as an important predictor of outcome in patients with T3a RCC 
with fat invasion alone according to the 2002 and 2009 AJCC TNM staging system, respectively. They proposed that a new tumor size cutoff $(7 \mathrm{~cm})$ parameter should be considered in the classification system for T3a and can yield the highest prediction accuracy. In our present study, we observed that the association of tumor size with prognosis risk was the non-linear shape. For patients with T3a RCC, a tumor size $>10 \mathrm{~cm}$ did not contribute to decrease the OS and CSS with the tumor size increases while increased rapidly between around $5 \mathrm{~cm}$ and $10 \mathrm{~cm}$. Therefore, tumor size in the future T3a staging system should be considered.

Although our study was vast in size and scope, the following limitations are presented: data was gathered from the SEER database and as such is retrospective. Moreover, information regarding diagnosis, symptoms, comorbidities, histopathologic features of tumor necrosis and lymphovascular invasion, metastatic patterns, lack of centralized pathological review, and the inability to differentiate types I and II papillary RCC, recurrence time, and recurrent disease treatment were not available and hence not analyzed. Potential selection bias also cannot be ruled out because patients with missing information (ethnicity, stage, and nuclear grade) were excluded. In addition, because the clinical incidence of T3a cases is not high, our study lacks verification of external data sets, but the prior published literature also supports our findings. Our conclusion is equivalent to the verification of the previous small sample size data. We also expect multi-center cooperation to solve this problem.

\section{Conclusion}

In our present study, we identified that the extrarenal invasion patterns and tumor size were two significant risk factors of OS and CSS for patients with T3a RCCs and increased the prognosis predictive ability when considered two covariables. From the results of our study and prior publications, it indicated that a proposed new modified T3a stage system is needed for the next revision of the TNM classification for RCC.

\section{Data Sharing Statement}

The data came from the Surveillance, Epidemiology, and End Results database (https://seer.cancer.gov/), which is public for all researches.

\section{Acknowledgments}

The authors thank the Surveillance, Epidemiology, and End Results (SEER) Program for kindly providing the clinical data. And we thank Bullet Edits Limited for the linguistic editing and proofreading of the manuscript.

\section{Author Contributions}

All authors made a significant contribution to the work reported, whether that is in the conception, study design, execution, acquisition of data, analysis, and interpretation, or in all these areas; took part in drafting, revising, or critically reviewing the article; gave final approval of the version to be published; have agreed on the journal to which the article has been submitted, and agree to be accountable for all aspects of the work.

\section{Funding}

There is no funding to report.

\section{Disclosure}

All authors declare that they have no competing interests.

\section{References}

1. Amin MB, Edge S, Greene F, et al. AJCC Cancer Staging Manual. Springer International Publishing; 2017.

2. Paner GP, Stadler WM, Hansel DE, Montironi R, Lin DW, Amin MB Updates in the eighth edition of the tumor-node-metastasis staging classification for urologic cancers. Eur Urol. 2018;73(4):560-569. doi:10.1016/j.eururo.2017.12.018

3. Novara G, Ficarra V, Antonelli A, et al. Validation of the 2009 TNM version in a large multi-institutional cohort of patients treated for renal cell carcinoma: are further improvements needed? Eur Urol. 2010;58(4):588-595. doi:10.1016/j.eururo.2010.07.006

4. Lam JS, Klatte T, Patard -J-J, et al. Prognostic relevance of tumour size in T3a renal cell carcinoma: a multicentre experience. Eur Urol. 2007;52(1):155-162. doi:10.1016/j.eururo.2007.01.106

5. Bedke J, Buse S, Pritsch M, et al. Perinephric and renal sinus fat infiltration in pT3a renal cell carcinoma: possible prognostic differences. BJU Int. 2009;103(10):1349-1354. doi:10.1111/j.1464410X.2008.08236.x

6. Margulis V, Tamboli P, Matin SF, Meisner M, Swanson DA, Wood CG. Location of extrarenal tumor extension does not impact survival of patients with pT3a renal cell carcinoma. $J$ Urol. 2007;178 (5):1878-1882. doi:10.1016/j.juro.2007.07.011

7. Wang Z, Yu K, Zhu Y, et al. Multiple patterns of perirenal fat invasion are associated with a poorer prognosis compared with isolated invasion: a proposal for a revision of T3aN0M0 TNM staging system. Front Oncol. 2020;10:336. doi:10.3389/fonc.2020.00336

8. Thompson RH, Leibovich $\mathrm{BC}$, Cheville JC, et al. Is renal sinus fat invasion the same as perinephric fat invasion for pT3a renal cell carcinoma? J Urol. 2005;174(4 Pt 1):1218-1221. doi:10.1097/01. ju.0000173942.19990.40

9. Bertini R, Roscigno M, Freschi M, et al. Renal sinus fat invasion in pT3a clear cell renal cell carcinoma affects outcomes of patients without nodal involvement or distant metastases. J Urol. 2009;181 (5):2027-2032. doi:10.1016/j.juro.2009.01.048

10. Poon SA, Gonzalez JR, Benson MC, McKiernan JM. Invasion of renal sinus fat is not an independent predictor of survival in pT3a renal cell carcinoma. BJU Int. 2009;103(12):1622-1625. doi:10.1111/ j.1464-410X.2008.08239.x 
11. Chen K, Lee BL, Huang HH, et al. Tumor size and Fuhrman grade further enhance the prognostic impact of perinephric fat invasion and renal vein extension in T3a staging of renal cell carcinoma. Int $J$ Urol. 2017;24(1):51-58. doi:10.1111/iju.13237

12. Kresowik TP, Johnson MT, Joudi FN. Combined renal sinus fat and perinephric fat renal cell carcinoma invasion has a worse prognosis than either alone. $J$ Urol. 2010;184(1):48-52. doi:10.1016/j. juro.2010.03.010

13. Shah PH, Lyon TD, Lohse CM, et al. Prognostic evaluation of perinephric fat, renal sinus fat, and renal vein invasion for patients with pathologic stage T3a clear cell renal cell carcinoma. BJU Int. 2018. doi:10.1111/bju.14523

14. da Costa WH, Moniz RR, da Cunha IW, Fonseca FP, Guimaraes GC, de Cassio Zequi S. Impact of renal vein invasion and fat invasion in pT3a renal cell carcinoma. BJU Int. 2012;109(4):544-548. doi:10.1111/j.1464-410X.2011.10366.x

15. Baccos A, Brunocilla E, Schiavina R, et al. Differing risk of cancer death among patients with pathologic T3a renal cell carcinoma: identification of risk categories according to fat infiltration and renal vein thrombosis. Clin Genitourin Cancer. 2013;11 (4):451-457. doi:10.1016/j.clgc.2013.05.006

16. Suer E, Baltaci S, Burgu B, Aydogdu O, Gogus C. Significance of tumor size in renal cell cancer with perinephric fat infiltration: is TNM staging system adequate for predicting prognosis?. Urol $J$ Winter. 2013;10(1):774-779.

17. Brookman-May SD, May M, Wolff I, et al. Evaluation of the prognostic significance of perirenal fat invasion and tumor size in patients with pT1-pT3a localized renal cell carcinoma in a comprehensive multicenter study of the CORONA project. Can we improve prognostic discrimination for patients with stage pT3a tumors? Eur Urol. 2015;67(5):943-951. doi:10.1016/j.eururo.2014.11.055
18. Yoo C, Song C, Hong JH, Kim CS, Ahn H. Prognostic significance of perinephric fat infiltration and tumor size in renal cell carcinoma. $J$ Urol. 2008;180(2):486-91; discussion 491. doi:10.1016/j. juro.2008.04.034

19. Li L, Shi L, Zhang J, Fan Y, Li Q. The critical impact of tumor size in predicting cancer special survival for T3aM0M0 renal cell carcinoma: a proposal of an alternative T3aN0M0 stage. Cancer Med. 2021;10(2):605-614. doi:10.1002/cam4.3629

20. Chevinsky M, Imnadze M, Sankin A, et al. Pathological stage T3a significantly increases disease recurrence across all tumor sizes in renal cell carcinoma. J Urol. 2015;194(2):310-315. doi:10.1016/j. juro.2015.02.013

21. Liu S, Feng C, Liu C, Wang Z. Comparison of prognosis between patients undergoing radical nephrectomy versus partial nephrectomy for renal cell carcinoma $\leq 7 \mathrm{~cm}$ T3aN0/xM0: survival benefit is biased toward partial nephrectomy. Cancer Med. 2021;10(24):8909-8923. doi:10.1002/cam4.4412

22. Ball MW, Gorin MA, Harris KT, et al. Extent of renal vein invasion influences prognosis in patients with renal cell carcinoma. BJU Int. 2016;118(1):112-117. doi:10.1111/bju.13349

23. Roos FC, Weirich J, Victor A, et al. Impact of several histopathological prognosticators and local tumour extension on oncological outcome in pT3b/c N0M0 renal cell carcinoma. BJU Int. 2009;104 (4):461-469. doi:10.1111/j.1464-410X.2009.08489.x

24. Mouracade P, Dagenais J, Chavali JS, et al. Perinephric and sinus fat invasion in stage pT3a tumors managed by partial nephrectomy. Clin Genitourin Cancer. 2017;16(5):e1077-e1082. doi:10.1016/j.clgc.2017. 07.019

25. Siemer S, Lehmann J, Loch A, et al. Current TNM classification of renal cell carcinoma evaluated: revising stage T3a. J Urol. 2005;173 (1):33-37. doi:10.1097/01.ju.0000146719.43269.e8
International Journal of General Medicine

\section{Publish your work in this journal}

The International Journal of General Medicine is an international, peer-reviewed open-access journal that focuses on general and internal medicine, pathogenesis, epidemiology, diagnosis, monitoring and treatment protocols. The journal is characterized by the rapid reporting of reviews, original research and clinical studies across all disease areas. The manuscript management system is completely online and includes a very quick and fair peer-review system, which is all easy to use. Visit http://www.dovepress.com/ testimonials.php to read real quotes from published authors. 